\title{
Implementation of uniform information on fetal movement in a Norwegian population reduced delayed reporting of decreased fetal movement and stillbirths in primiparous women - a clinical quality improvement
}

Eli Saastad ${ }^{1,2,4^{*}}$, Julie Victoria Holm Tveit ${ }^{3,4}$, Vicki Flenady ${ }^{5}$, Babill Stray-Pedersen ${ }^{3,4}$, Ruth C Fretts ${ }^{6}$, Per E Børdahl ${ }^{7}$, J Frederik Frøen'

\begin{abstract}
Background: Delayed maternal reporting of decreased fetal movement (DFM) is associated with adverse pregnancy outcomes. Inconsistent information on fetal activity to women during the antenatal period may result in delayed reporting of DFM. We aimed to evaluate an intervention of implementation of uniform information on fetal activity to women during the antenatal period.

Methods: In a prospective before-and-after study, singleton women presenting DFM in the third trimester across 14 hospitals in Norway were registered. Outcome measures were maternal behavior regarding reporting of DFM, concerns and stillbirth. In addition, cross-sectional studies of all women giving birth were undertaken to assess maternal concerns about fetal activity, and population-based data were obtained from the Medical Birth Registry Norway.

Results: Pre- and post-intervention cohorts included 19407 and 46143 births with 1215 and 3038 women with DFM respectively. Among primiparous women with DFM, a reduction in delayed reporting of DFM ( $\geq 48 \mathrm{hrs}) \mathrm{OR}$ 0.61 (95\% Cl 0.47-0.81) and stillbirths OR 0.36 (95\% Cl 0.19-0.69) was shown in the post-intervention period. No difference was shown in rates of consultations for DFM or maternal concerns. Stillbirth rates and maternal behavior among women who were of non-Western origin, smokers, overweight or $>34$ years old were unchanged.

Conclusions: Uniform information on fetal activity provided to pregnant women was associated with a reduction in the number of primiparous women who delayed reporting of DFM and a reduction of the stillbirth rates for primiparous women reporting DFM. The information did not appear to increase maternal concerns or rate of consultation. Due to different imperfections in different clinical settings, further studies in other populations replicating these findings are required.
\end{abstract}

\section{Background}

Women presenting with decreased fetal movement (DFM) are at increased risk of fetal growth restriction, stillbirth, preterm birth and emergency caesarean section [1-5]. Excessive delay in maternal reporting of DFM is associated with perinatal deaths $[5,6]$. There is no

\footnotetext{
* Correspondence: eli.saastad@fhi.no

${ }^{1}$ Norwegian Institute of Public Health, Division of Epidemiology, Oslo, Norway
}

\section{Biomed Central}

agreement on any quantitative limit between "normal" versus "abnormal" fetal activity $[7,8]$, due to normal variation among healthy fetuses [9] and variation in maternal ability to perceive fetal activity [10]. The only definition of DFM based on focused counting data in a total population, is the rule of " 10 fetal movements within two hours", which subsequently has been tested as a screening tool $[7,11]$. Fetal movement counting (FMC) is a method used by the mother to quantify her 
baby's movements. Various methods with different alarm limits have been published; discussed elsewhere $[7,8]$. FMC is not promoted as a universal screening tool for fetal wellbeing [4], but has been recommended in high-risk pregnancies $[12,13]$.

The most important clinical screening tool for DFM for identifying high-risk pregnancies is the women's own perception of a decrease [8,14-16]; i.e. her perception of a change, not the crossing of a given limit. Existing guidelines for antenatal care in the United Kingdom, the US and Norway recommend that a distinct reduction of fetal movement should be reported and lead to further investigation [17-20]. In our Norwegian setting nearly $100 \%$ of all pregnant women attend the public antenatal care program provided by community midwives and general practitioners. Pregnant women with a concern of DFM usually contact maternity wards directly. Four to fifteen percent of women present to the hospital in late pregnancy with the primary complaint of reduced or absent fetal movements $[8,21,22]$.

The current study was a part of the ongoing, interdisciplinary collaborative effort related to DFM: Fetal Movement Intervention Assessment (Femina), aiming to survey clinical management and initiate quality improvement efforts in Australia \& New Zealand [23], the US [24], the United Kingdom [15] and Norway. The information pamphlet provided to expectant mothers by Norwegian health authorities, instructs women to contact a midwife or a physician "if the baby has become very calm, if they feel less movements - a few or no movements from the fetus" $[18,20]$. In Norway, significant variation has been shown in maternal recall of information received about fetal movement [10]. Further, women who waited $>24$ hours with reduced or absent movement before contacting healthcare have been shown to be at increased risk for adverse outcomes [22]. Maternal recall of having received information about fetal movement was associated with more frequent concerns, without improving pregnancy outcomes [10].

Variation in clinical practice, as reflected in patient information, may represent increased risk [25]. Quality assurance efforts aimed at health providers (through clinical guidelines) and pregnant women (through uniform information) were implemented in order to increase identification of high-risk pregnancies for optimal observation and treatment. This paper reports the effects of providing uniform information about fetal activity on maternal awareness, behavior, concerns and pregnancy outcomes when DFM was perceived by the mothers. We hypothesised that providing this information would reduce the number of women who delayed reporting DFM to their healthcare provider, in the total population or by the subgroups defined by maternal age $[5,26]$, body mass index (BMI) $[5,27]$, smoking habits
[5,28], and maternal country of origin [29]. We also hypothesised that the intervention was associated with improved pregnancy outcomes, overall and/or by the subgroups. The guidelines for health care providers and effects on clinical management are presented elsewhere [30].

\section{Methods}

\section{The intervention - information on fetal activity and monitoring}

Due to limited high level evidence, the brochure of information was developed using a consensus-based approach; by a systematic literature review, and consultation with leading academics in midwifery and obstetrics across all participating hospitals and a group of pregnant women. The brochure, which included a fetal movement chart (a kick chart), was provided at the ultrasound screening assessment in gestational week 1719 , which $98 \%$ of the women attend. The brochure covered information on: expected normal fetal activity [31]; differences in perception according to different fetal movements [31], maternal position [32], the inter- and intraindividual variation between fetuses [9], maternal weight [27], and smoking [33]; interpretation of variation of fetal activity; instructions on how to use the kick chart; and when to contact health professionals if experiencing DFM [11].

Women were informed that their subjective assessment of a decrease in fetal activity was the most important marker of DFM - taking priority over any formal DFM alarm limits [8]. They were instructed not to wait until the next day if they perceived complete absence of fetal activity or if they felt a significant and sustained decrease. If in doubt, as a "thumb rule", they were advised in accordance with the most validated definition for focused counting $[11,34]$ : that a healthy baby very rarely has less than 10 movement in the course of two hours when it usually is active [35]. The brochure was available in Norwegian (Additional file 1), English (Additional file 2), Urdu (Additional file 3), Somali (Additional file 4), Turkish (Additional file 5) and Arabic (Additional file 6). The kick chart was suggested as a supportive tool for women who wished to use it. A modified "count-to-ten" chart $[11,36]$ was chosen, as this has the highest compliance and acceptance rates $[4,37,38]$. Use of a kick chart is exemplified in additional file 7.

To assist in the clinicians' implementation of this brochure, written information and newsletters were distributed to participating hospitals and regular meetings between clinicians and the study staff were arranged.

\section{Data collection}

Fourteen hospitals across both urban and rural districts, with a total of approximately 33, 000 births annually, 
were included in the before-and-after study. Two different data collection methods were used pre- and postintervention: 1) Prospective data collection for women presenting with DFM (DFM population), and 2) Crosssectional studies (Cross-sectional population):

1) Prospective data collection for all women with singleton pregnancies of $\geq 28$ weeks of gestation presenting at the hospital with a concern of DFM was undertaken by the caregiver without maternal consent and forwarded as anonymous data to the study coordinating centre. Data were collected on maternal demographic characteristics, delay in reporting DFM, clinical management of DFM and pregnancy outcome. Following baseline data collection over a seven month period from April to October 2005, post-intervention data were collected for the 16 month period from November 2005 to March 2007.

2) Cross-sectional studies were performed; pre-intervention (June 2005) and post-intervention (February 2007). Women who birthed at one of the participating hospitals completed a survey anonymously prior to hospital discharge. Further description of this data collection is presented elsewhere [10]. The sample size for the cross-sectional studies was weighted according to number of births in the respective hospitals during the study period.

In addition, population-based data were obtained from the Medical Birth Registry Norway [39] for the purpose of comparisons of the covariates in the study populations versus the total population deliveries in the area. The studies were approved by The Regional Committees for Medical Research Ethics and The Norwegian Data Inspectorate.

\section{Outcome measures}

\section{Primary outcome measure}

The primary outcome measure was maternal behavior in relation to reporting perceived absence or decreased fetal movement to the health provider; defined as the rate of women waiting $\geq 25$ hours with absent fetal movement or $\geq 48$ hours with DFM $[6,16,29,40,41]$.

\section{Secondary outcome measures}

- Maternal awareness: maternal self-report of attention paid to fetal activity.

- Maternal concerns: maternal self-report of the frequency of concerns about DFM; dichotomized into being concerned "twice or more" versus "once or never".

- Receiving information: maternal self-report of receiving information about fetal activity.

- DFM consultation: a consultation at the hospital because of maternal perception of DFM.
- Pregnancy outcome for women with DFM was stillbirth; and, for the cross-sectional population; small for gestational (SGA) $<10^{\text {th }}$ centile (customized) [42] and emergency cesarean section.

- Counting group: proportion of women reporting using a kick chart more than once per week.

Effectiveness in distribution of information and maternal internalization of information were assessed by combining cross-sectional data with the stillbirth rate at hospital levels. As a proxy for effectiveness in distribution, we compared the hospital specific percentage of women reporting receipt of the written information from the cross-sectional surveys with the stillbirth rate in the DFM population. As a proxy for internalization of the information, the percentage for women reporting having used the kick chart twice a week or more was compared with the stillbirth rate in the DFM population.

\section{Analyses}

Statistical analyses were performed in SPSS 14.0.1 (SPSS Inc., Chicago, IL). Crude and adjusted odds ratios (ORs) with 95\% confidence intervals (CIs) were estimated, and variables with associations with a $\mathrm{p}<0.20$ in univariate analyses were included in the multivariate models [43]. Chi square tests were used for estimating differences between proportions of categorical variables. A p-value $<0.05$ was considered statistically significant. Bonferroni corrections were performed in the multiple comparisons. Subgroup analyses were undertaken according to: maternal age $[5,26]$, body mass index (BMI) $[5,27]$, smoking habits $[5,28]$, and maternal country of origin [29] and according to subgroups of Western and nonWestern origin (due to higher rates of stillbirths among non-Western women in our community) [29]. Western mothers were defined as women with origin in Western Europe, North America and Oceania. For women with more than one episode of reporting DFM, only the first episode was included in the analyses.

\section{Results}

Overview data collection is presented in Figure 1. Baseline characteristics of the populations are described in Table 1 . The respondents in the cross-sectional studies were representative for the pregnant population in their area during the study period in regard to age, parity and smoking habits (data from the Medical Birth Registry Norway, not shown).

\section{Information and maternal awareness of fetal activity}

Data from the cross-sectional studies showed that one in four women did not recall receiving information about normal expected fetal activity by their health provider, both pre- and post-intervention. Recall of 
Table 1 Descriptive characteristics: DFM and Cross-sectional populations

\begin{tabular}{|c|c|c|c|c|c|c|}
\hline \multirow[b]{2}{*}{ Characteristics } & \multicolumn{2}{|c|}{$\begin{array}{c}\text { DFM* } \\
\mathrm{N}=4253 \\
\end{array}$} & \multicolumn{4}{|c|}{$\begin{array}{c}\text { Cross-sectional* } \\
\mathrm{N}=1431\end{array}$} \\
\hline & $\begin{array}{r}\text { Pre-intervention } \\
n=1215 \\
n(\%) \dagger\end{array}$ & $\begin{array}{r}\text { Post-intervention } \\
\mathrm{n}=3038 \\
\mathrm{n}(\%) \dagger\end{array}$ & $P \neq$ & $\begin{array}{r}\text { Pre-intervention } \\
n=692 \\
n(\%) \dagger\end{array}$ & $\begin{array}{r}\text { Post-intervention } \\
\mathrm{n}=739 \\
\mathrm{n}(\%) \dagger\end{array}$ & $P \neq$ \\
\hline Age, y mean (SD) & $29.6(4.9)$ & $29.7(5.2)$ & 0.625 & $30.2(4.9)$ & $30.1(5.1)$ & 0.849 \\
\hline$<20$ & $23(1.9)$ & $59(2.0)$ & & $9(1.3)$ & $10(1.4)$ & \\
\hline $20-24$ & $182(15.1)$ & $454(15.1)$ & & $70(10.3)$ & $101(13.7)$ & \\
\hline $25-29$ & $388(32.3)$ & $933(31.1)$ & & $231(34.0)$ & $208(28.1)$ & \\
\hline $30-34$ & $413(34.4)$ & 1031 (34.3) & & $237(34.9)$ & $273(36.9)$ & \\
\hline $35+$ & $196(16.3)$ & 527 (17.5) & & 133 (19.6) & 147 (19.9) & \\
\hline \multicolumn{7}{|l|}{ Parity } \\
\hline Para 0 & $559(51.1)$ & $1414(52.4)$ & 0.490 & $287(43.1)$ & $300(41.4)$ & 0.197 \\
\hline Para 1 & $372(34.0)$ & 878 (32.5) & & $221(33.2)$ & $283(39.0)$ & \\
\hline Para 2+ & $163(14.9)$ & 409 (15.2) & & $158(23.7)$ & $142(19.5)$ & \\
\hline $\mathrm{BMI}, \mathrm{kg} / \mathrm{m}^{2}$ & $24.7(5.1)$ & $24.5(5.0)$ & 0.547 & $24.4(4.4)$ & $23.6(4.2)$ & $<0.001$ \\
\hline$<20$ & $143(13.3)$ & $383(14.2)$ & & $74(11.0)$ & $113(15.6)$ & \\
\hline $20-24$ & $547(50.8)$ & $1325(49.0)$ & & $378(56.2)$ & $412(56.9)$ & \\
\hline $25-29$ & $244(22.7)$ & 638 (23.6) & & $147(21.8)$ & 137 (18.9) & \\
\hline $30+$ & $91(8.5)$ & $249(9.2)$ & & $74(11.0)$ & $21(8.6)$ & \\
\hline \multicolumn{7}{|l|}{ Smoking habits } \\
\hline Smoking & $104(8.8)$ & 259 (8.9) & 0.924 & $50(7.4)$ & $48(6.4)$ & 0.483 \\
\hline \multicolumn{7}{|l|}{ Country of origin } \\
\hline Non-Western & $178(14.7)$ & $406(13.4)$ & 0.271 & $39(5.7)$ & $29(3.6)$ & 0.064 \\
\hline
\end{tabular}

* Data are reported as $\mathrm{n}(\%)$ unless otherwise noted.

† Denominators vary due to missing values

₹ Chi square tests for the difference between proportions within women with DFM and the cross-sectional population respectively

receiving information was associated with higher awareness of fetal activity, both pre-intervention (OR 2.0, 95\% CI 1.2-3.3) and post-intervention (OR 1.8, 95\% CI 1.0$3.1, \mathrm{p}=0.043)$. Pre-intervention, recall of receiving information was associated with more frequent maternal concern (OR 1.7, 95\% CI 1.2-2.4); while this association was not longer present post-intervention (OR 1.3, 95\% CI 0.9-1.9).

Maternal recall of information about limits for normality was more homogeneous in the intervention period, e.g. $22 \%$ recalled having seen the thumb rule (10 kicks in two hours) at baseline measurement, versus $42 \%$ in the intervention period ( $\mathrm{p}=0.022$ ). Pre-intervention, low maternal awareness to fetal activity was associated with an increased risk of having an SGA baby; [10] this association was not observed in the post-intervention period (OR 1.3, 95\% CI 0.6-2.9).

\section{Maternal behavior and pregnancy outcomes}

Among women with DFM, the stillbirth rate was lower in post-intervention period; $4.2 \%$ versus $2.4 \%$ (Tveit et al, submitted 2009). The reduction in stillbirth was isolated to primiparous women only. Primiparous women also reported DFM earlier than all other women included (Table 2). In the total population, the mean gestational age at the time of reporting DFM was two days lower during the post-intervention period; $36^{6}$ versus $36^{4}$ weeks, $\mathrm{p}=0.006$.

In the post-intervention group, overweight women in the cross-sectional populations described higher awareness of fetal activity (Table 3 ). No behavior changes were observed among overweight women if they perceived DFM (Table 2).

Pre-intervention, smoking mothers in the cross-sectional population recalled less receipt of information about fetal activity than non-smokers, OR 0.5 (95\% CI $0.3-0.9)$. This association was not present in post-intervention, OR 0.6 (95\% CI 0.3-1.2). No changes in maternal behavior were observed among smoking women perceiving DFM (Table 2).

Non-Western women in the cross-sectional study post-intervention, remained the only risk group reporting both less receipt of information (adjusted OR 0.4, 95\% CI 0.2-0.8) and low awareness of fetal activity (Table 3). Among the non-Western women who perceived DFM, the intervention showed no changes in maternal behavior, frequency of concerns or outcomes (Table 2).

The hospital-specific percentage of women reporting having received written information (proxy for 


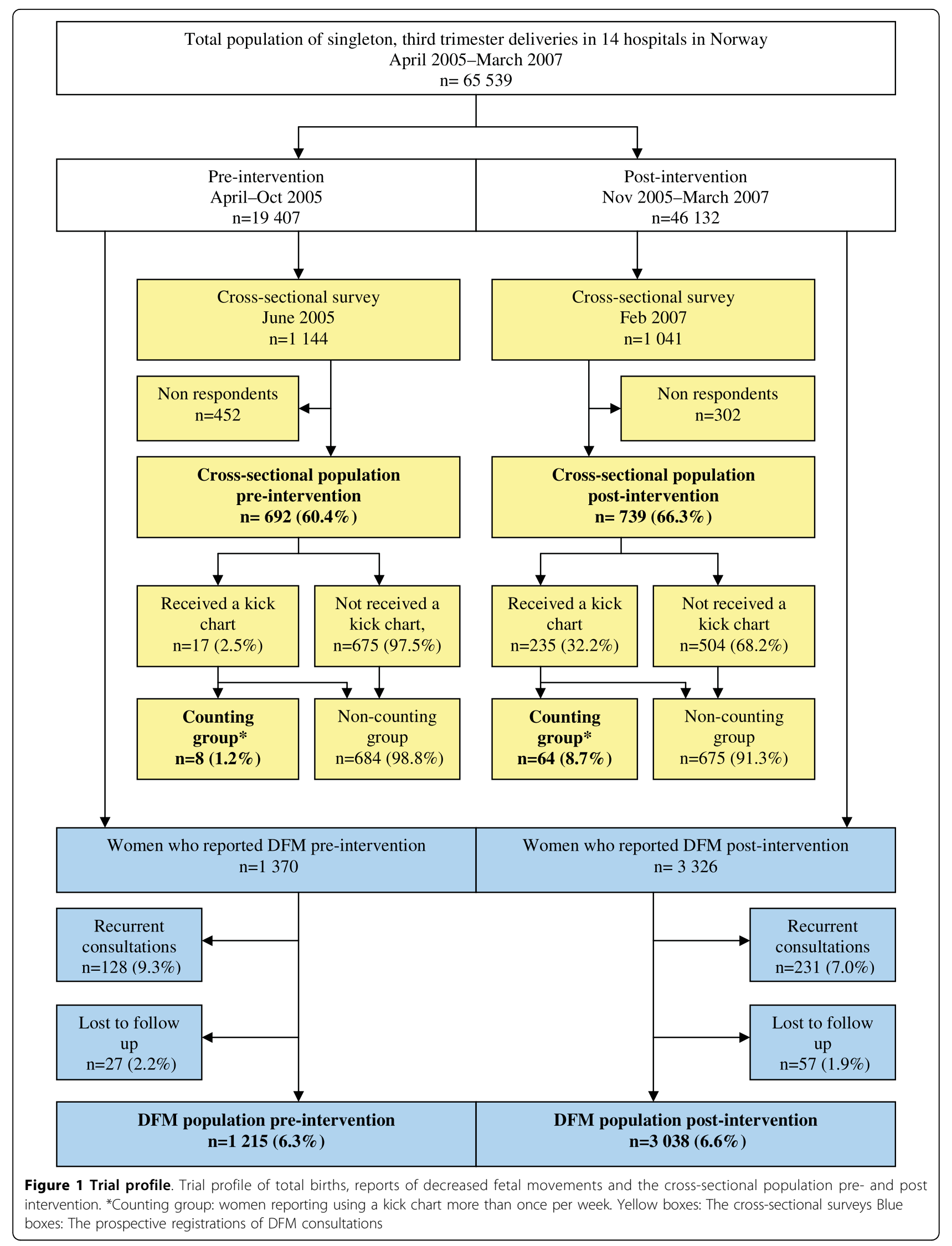


Table 2 DFM population: Effects of intervention on maternal behavior and stillbirth rates, stratified by subgroups

\begin{tabular}{|c|c|c|c|c|c|c|c|c|c|c|c|c|}
\hline & \multicolumn{2}{|c|}{$\begin{array}{c}\text { Overall } \\
N=4253\end{array}$} & \multicolumn{10}{|c|}{$\begin{array}{c}\text { Stratified by subgroups } \\
\mathrm{n}(\%)\end{array}$} \\
\hline & $\begin{array}{r}\text { Pre- } \\
\text { intervention } \\
\mathrm{n}=1215 \\
\mathrm{n}(\%)\end{array}$ & $\begin{array}{r}\text { Post- } \\
\text { intervention } \\
\mathrm{n}=3038 \\
\mathrm{n}(\%)\end{array}$ & $\begin{array}{r}\text { Primiparous } \\
\mathrm{n}=1973 \\
(52.0)\end{array}$ & $\begin{array}{l}\mathrm{P}- \\
\text { value }\end{array}$ & $\begin{array}{r}\geq 35 \\
\text { years } \\
n= \\
724 \\
(17.2)\end{array}$ & $\begin{array}{r}P- \\
\text { value }\end{array}$ & $\begin{array}{r}\text { Overweight } \\
\mathrm{n}=1400 \\
(36.8)\end{array}$ & $\begin{array}{l}P- \\
\text { value }\end{array}$ & $\begin{array}{r}\text { Smokers } \\
\mathrm{n}=363 \\
(8.9)\end{array}$ & $\begin{array}{l}P- \\
\text { value }\end{array}$ & $\begin{array}{r}\text { Non- } \\
\text { Western } \\
\mathrm{n}=583 \\
(13.7)\end{array}$ & $\begin{array}{r}\mathrm{P}- \\
\text { value }\end{array}$ \\
\hline Consultation & & & \multicolumn{10}{|c|}{ Adj OR $(95 \% \mathrm{Cl})^{5}$} \\
\hline $\begin{array}{l}\text { By own } \\
\text { initiative }\end{array}$ & $363(32.1)$ & $656(30.8)$ & $\begin{array}{r}1.22 \\
(0.95-1.57)\end{array}$ & 0.117 & $\begin{array}{r}1.43 \\
(0.99- \\
2.08)\end{array}$ & 0.652 & $\begin{array}{r}1.10 \\
(0.81-1.50)\end{array}$ & 0.524 & $\begin{array}{r}0.87 \\
(0.47- \\
1.62)\end{array}$ & 0.657 & $\begin{array}{r}0.88 \\
(0.54- \\
1.46)\end{array}$ & 0.631 \\
\hline $\begin{array}{l}\text { At the } \\
\text { delivery unit }\end{array}$ & $661(54.9)$ & $1,716(57.4)$ & $\begin{array}{r}1.35 \\
(1.09-1.67)\end{array}$ & 0.007 & $\begin{array}{r}1.01 \\
(0.76- \\
1.34)\end{array}$ & 0.057 & $\begin{array}{r}1.24 \\
(0.96-1.60)\end{array}$ & 0.106 & $\begin{array}{r}0.82 \\
(0.47- \\
1.44)\end{array}$ & 0.493 & $\begin{array}{r}1.06 \\
(0.68- \\
1.67)\end{array}$ & 0.786 \\
\hline $\begin{array}{l}\text { During night } \\
\text { (6 pm-8 am) }\end{array}$ & $317(27.7)$ & 846 (29.9) & $\begin{array}{r}1.39 \\
(1.09-1.77)\end{array}$ & 0.007 & $\begin{array}{r}1.31 \\
(0.83- \\
2.07)\end{array}$ & 0.258 & $\begin{array}{r}1.41 \\
(1.05-1.89)\end{array}$ & 0.023 & $\begin{array}{r}1.78 \\
(0.99- \\
3.21)\end{array}$ & 0.055 & $\begin{array}{r}1.08 \\
(0.65- \\
1.80)\end{array}$ & 0.775 \\
\hline In weekends & $258(21.2)$ & $607(20.0)$ & $\begin{array}{r}1.04 \\
(0.80-1.35)\end{array}$ & 0.758 & $\begin{array}{r}0.84 \\
(0.65- \\
1.69)\end{array}$ & 0.855 & $\begin{array}{r}1.28 \\
(0.94-1.76)\end{array}$ & 0.121 & $\begin{array}{r}0.51 \\
(0.27- \\
0.96)\end{array}$ & 0.037 & $\begin{array}{r}0.88 \\
(0.50- \\
1.52)\end{array}$ & 0.634 \\
\hline DFM $\geq 48$ hrs & 415 (53.6) & $897(48.9)$ & $\begin{array}{r}0.61 \\
(0.47-0.81)\end{array}$ & $<0.001$ & $\begin{array}{r}0.82 \\
(0.51- \\
1.32)\end{array}$ & 0.414 & $\begin{array}{r}1.12 \\
(0.81-1.54)\end{array}$ & 0.507 & $\begin{array}{r}1.47 \\
(0.74- \\
2.91)\end{array}$ & 0.274 & $\begin{array}{r}0.54 \\
(0.29- \\
0.99)\end{array}$ & 0.045 \\
\hline $\begin{array}{l}\text { Absent FM } \geq \\
25 \text { hrs }\end{array}$ & 99 (23.9) & 201 (18.0) & $\begin{array}{r}0.72 \\
(0.47-1.09)\end{array}$ & 0.117 & $\begin{array}{r}1.00 \\
(0.43- \\
2.32)\end{array}$ & 0.996 & $\begin{array}{r}0.90 \\
(0.55-1.47)\end{array}$ & 0.668 & $\begin{array}{r}0.60 \\
(0.22- \\
1.61)\end{array}$ & 0.309 & $\begin{array}{r}0.64 \\
(0.29- \\
1.43)\end{array}$ & 0.274 \\
\hline Fetal deaths & $50(4.2)$ & $73(2.4)$ & $\begin{array}{r}0.36 \\
(0.19-0.69)\end{array}$ & 0.002 & $\begin{array}{r}0.92 \\
(0.35- \\
2.44)\end{array}$ & 0.902 & $\begin{array}{r}0.60 \\
(0.30-1.20)\end{array}$ & 0.151 & $\begin{array}{r}1.48 \\
(0.40- \\
5.53)\end{array}$ & 0.559 & $\begin{array}{r}0.99 \\
(0.25- \\
4.02)\end{array}$ & 0.993 \\
\hline
\end{tabular}

${ }^{\dagger}$ Univariate logistic regression analyses with $95 \% \mathrm{Cl}$, at baseline is the reference category

$\S$ Multivariate logistic regression analyses with $95 \% \mathrm{Cl}$, adjusting for the covariates (parity, maternal age, BMI, smoking habits, maternal origin) Denominators vary due to missing values. Bold numbers indicate significant values after Bonferroni correction for multiple comparisons

distribution) was negatively associated with mortality rates - the more information, the lower mortality $(\beta=$ $0.974, \mathrm{p}=0.031$ ). This was done to assess the effect of the distribution of information and maternal internalization of it on the number of stillbirths.

\section{Maternal concerns - as reported by women in the cross- sectional studies}

Mothers in the post-intervention period did not report concerns or have a DFM consultation more frequently (Table 4). Overweight women were the only subgroup reporting increased concerns; however, this was not significant after Bonferroni correction (Table 4). When concerned, the mothers more often related their concern to the fetal activity level earlier in the actual pregnancy ( $44 \%$ vs. $51 \%, p=0.011$ ). More often, the concerned mothers assessed their perception of DFM not being normal for their baby and that their concern was a true reason for being concerned ( $28 \%$ vs. $33 \%, \mathrm{p}=0.022$ ). Being concerned was associated with being examined at hospital both pre-intervention (OR 4.9, 95\% CI 3.0-7.8) and post-intervention (OR 5.8, 95\% CI 3.7-9.2).
Fetal movement counting in the intervention group In the post-intervention group, 235 (32\%) reported using a kick chart, as opposed to 8 (1\%) pre-intervention. Post-intervention, 64 (9\%) of women used a kick chart more than once per week (counting group); versus 8 (1\%) pre-intervention. Primiparous women were more likely than multiparous women to use a kick chart more than once per week (OR 2.3, 95\% CI 1.3-4.2). No nonWestern mothers used a kick chart.

Maternal experiences with use of a kick chart in the intervention period are presented in Table 5, illustrating the benefits of maternal receipt of receiving information on how and why to use the kick chart. The use of a kick chart was not associated with increased maternal concerns about DFM (32\% in the non-counting group vs. $42 \%$ in the counting group, $\mathrm{p}=0.090)$. Use of a kick chart was associated with a reduced risk of having a DFM consultation, $18 \%$ vs. $9 \%(\mathrm{p}=0.045)$. One of ten babies was SGA in both groups. Eleven (7\%) of the non-counting group had an emergency caesarean section, as opposed to one (2\%) in the counting group $(\mathrm{p}=0.047)$. 
Table 3 Cross-sectional population: Low maternal awareness of fetal activity and maternal characteristics $(\mathrm{N}=1431)^{*}$

\begin{tabular}{|c|c|c|c|c|c|c|}
\hline \multirow[b]{3}{*}{ Maternal characteristics } & \multicolumn{3}{|c|}{ Pre-intervention, $\mathrm{n}=692$} & \multicolumn{3}{|c|}{ Post-intervention, $n=739$} \\
\hline & \multicolumn{4}{|c|}{$\begin{array}{c}\text { Low maternal awareness } \dagger \\
\mathrm{n}=78(11.7 \%)\end{array}$} & \multicolumn{2}{|c|}{$\begin{array}{l}\text { Low maternal awareness } \dagger \\
n=62(8.9 \%)\end{array}$} \\
\hline & $\begin{array}{r}\text { Values } \\
\mathrm{n}(\%)\end{array}$ & $\begin{array}{r}\text { Crude OR } \\
(95 \% \mathrm{Cl}) \\
\end{array}$ & $\begin{array}{r}\text { Adj OR } \\
(95 \% \mathrm{Cl}) \\
\end{array}$ & $\begin{array}{r}\text { Values } \\
\mathrm{n}(\%)\end{array}$ & Crude OR (95\% CI) & $\begin{array}{r}\text { Adj OR } \\
(95 \% \mathrm{Cl}) \\
\end{array}$ \\
\hline $\begin{array}{l}\text { Primiparous } \\
\text { (reference: multiparous) }\end{array}$ & $287(43.1)$ & $\begin{array}{r}0.57(0.34-0.96) \\
p=0.032\end{array}$ & $\begin{array}{r}0.87(0.58-1.30) \\
p=0.494\end{array}$ & $300(41.4)$ & $\begin{array}{r}0.98(0.57-1.67) \\
p=0.930\end{array}$ & Not included \\
\hline $\begin{array}{l}\text { Age } \geq 35 \text { yrs } \\
\text { (reference: }<35 \text { years old) }\end{array}$ & $133(19.6)$ & $\begin{array}{r}2.67(1.61-4.45) \\
p<0.001\end{array}$ & $\begin{array}{r}1.64(1.06-2.54) \\
p=0.026\end{array}$ & $147(19.9)$ & $\begin{array}{r}1.38(0.76-2.51) \\
p=0.290\end{array}$ & $\begin{array}{r}1.34(0.74-2.52) \\
p=0.316\end{array}$ \\
\hline $\begin{array}{l}\mathrm{BMI}>25 \mathrm{~kg} / \mathrm{m}^{2} \\
\text { (reference: } \mathrm{BMl} \leq 25 \mathrm{~kg} / \mathrm{m}^{2} \text { ) }\end{array}$ & $221(32.8)$ & $\begin{array}{r}1.38(0.84-2.27) \\
p=0.208\end{array}$ & $\begin{array}{r}0.77(0.50-1.18) \\
p=0.226\end{array}$ & $158(27.5)$ & $\begin{array}{r}0.53(0.27-1.04) \\
p=0.063\end{array}$ & $\begin{array}{r}0.43(0.21-0.89) \\
p=0.024\end{array}$ \\
\hline $\begin{array}{l}\text { Smokers } \\
\text { (reference: non-smokers) }\end{array}$ & $50(7.4)$ & $\begin{array}{r}0.67(0.24-2.00) \\
p=0.503\end{array}$ & Not included & $48(6.4)$ & $\begin{array}{r}0.74(0.22-2.46) \\
p=0.622\end{array}$ & Not included \\
\hline $\begin{array}{l}\text { Non-Western origin } \\
\text { (reference: Western origin) }\end{array}$ & $39(5.7)$ & $\begin{array}{r}2.54(1.11-5.83) \\
p=0.023\end{array}$ & $\begin{array}{r}1.79(0.83-3.83) \\
p=0.226\end{array}$ & $29(3.6)$ & $\begin{array}{r}3.50(1.34-9.08) \\
p=0.006\end{array}$ & $\begin{array}{r}3.34(1.27-8.78) \\
p=0.015\end{array}$ \\
\hline
\end{tabular}

* Detailed results from the baseline population are presented elsewhere [10]

+ Univariate and multivariate logistic regression analyses with $95 \% \mathrm{Cl}$ for the associations between the analyzed groups. Denominators vary due to missing values. Bold numbers indicate significant values.

The hospital-specific percentage of women reporting having used the kick chart more than once per week or more (proxy for internalization) was negatively associated with mortality $(\beta=0.922, \mathrm{p}=0.005)$. This does not reflect the effect of kick counting on an individual level, as there are no data to support this, only the benefit of effective information.

\section{Discussion}

In this prospective before-and-after study, primiparous women were shown to have the greatest behavioral change in reporting DFM and were the only risk group with a reduction in stillbirth. This may be associated with the experience of transition to the motherhood role of first-time mothers. With no previous experiences, pregnancy represents a major adjustment period, strongly influenced by information seeking and trying to adopt best health practices and changes in lifestyle [44].

While the effect of printed educational materials as guidelines for health care providers is associated with some improvement in process of care [45], the addition of additional interventions such as outreach education and audit and feedback may enhance this effect [46]. In this study, implementation of standardized information for women across participating hospitals was achieved through a multifaceted intervention including clinical practice recommendations, outreach education and audit and feedback. Standardized written information improved maternal self-screening of significance for

Table 4 Cross-sectional population: Effects of intervention on maternal awareness, concern and maternal behavior ( $\mathrm{N}$ = 1431)

\begin{tabular}{|c|c|c|c|c|c|c|c|c|c|}
\hline & \multicolumn{4}{|c|}{$\begin{array}{c}\text { Overall } \\
\mathrm{N}=1431 \\
\end{array}$} & \multicolumn{5}{|c|}{$\begin{array}{l}\text { Stratified by subgroups post-intervention, } n=739 \\
n(\%)^{*}\end{array}$} \\
\hline & $\begin{array}{r}\text { Pre- } \\
\text { intervention } \\
n=692 \\
n(\%)^{*}\end{array}$ & $\begin{array}{r}\text { Post- } \\
\text { intervention } \\
\mathrm{n}=715 \\
\mathrm{n}(\%)^{*}\end{array}$ & $\begin{array}{l}\text { Crude OR } \\
(95 \% \mathrm{Cl})^{+}\end{array}$ & $\begin{array}{r}\text { Adjusted } \\
\text { OR } \\
(95 \% \mathrm{Cl})^{\S}\end{array}$ & $\begin{array}{r}\text { Primiparous } \\
\mathrm{n}=587 \\
(57.8)\end{array}$ & $\begin{array}{r}\geq 35 \text { years } \\
\mathrm{n}=280 \\
(19.7)\end{array}$ & $\begin{array}{r}\text { Overweight } \\
\mathrm{n}=421 \\
(30.1)\end{array}$ & $\begin{array}{r}\text { Smokers } \\
\mathrm{n}=97(6.9)\end{array}$ & $\begin{array}{r}\text { Non- } \\
\text { Western } \\
\mathrm{n}=67(4.7)\end{array}$ \\
\hline & & & & & \multicolumn{5}{|c|}{ Adj OR $(95 \% \mathrm{Cl})^{\S}$} \\
\hline $\begin{array}{l}\text { Low } \\
\text { awareness }\end{array}$ & $78(11.7)$ & $62(8.9)$ & $\begin{array}{r}0.72(0.50- \\
1.02) \\
p=0.060\end{array}$ & $\begin{array}{r}0.84(0.57- \\
1.24) \\
p=0.356\end{array}$ & $\begin{array}{r}1.19(0.63- \\
2.26) \\
p=0.585\end{array}$ & $\begin{array}{r}0.49(0.24- \\
1.00) \\
p=0.050\end{array}$ & $\begin{array}{r}0.44(0.20- \\
1.00) \\
p=0.051\end{array}$ & $\begin{array}{r}0.74(0.15- \\
3.68) \\
p=0.712\end{array}$ & $\begin{array}{r}1.10(0.23- \\
5.27) \\
p=0.909\end{array}$ \\
\hline Concerned & $341(50.7)$ & $417(57.9)$ & $\begin{array}{r}1.15(0.93- \\
1.42) \\
p=0.210\end{array}$ & $\begin{array}{r}1.20(0.96- \\
1.50) \\
p=0.114\end{array}$ & $\begin{array}{r}0.95(0.68- \\
1.34) \\
p=0.783\end{array}$ & $\begin{array}{r}1.32(0.80- \\
2.19) \\
p=0.273\end{array}$ & $\begin{array}{r}1.54(1.03- \\
2.31) \\
p=0.037\end{array}$ & $\begin{array}{r}0.98(0.42- \\
2.34) \\
p=0.969\end{array}$ & $\begin{array}{r}3.21(1.04- \\
9.93) \\
p=0.043\end{array}$ \\
\hline $\begin{array}{l}\text { DFM } \\
\text { consultation }\end{array}$ & $98(14.2)$ & $122(16.4)$ & $\begin{array}{r}1.18(0.88- \\
1.57) \\
p=0.263\end{array}$ & $\begin{array}{r}1.32(0.97- \\
1.78) \\
p=0.075\end{array}$ & $\begin{array}{r}1.38(0.88- \\
2.17) \\
p=0.163\end{array}$ & $\begin{array}{r}1.10(0.54- \\
2.21) \\
p=0.801\end{array}$ & $\begin{array}{r}1.06(0.93- \\
2.63) \\
p=0.092\end{array}$ & $\begin{array}{r}1.32(0.46- \\
3.80) \\
p=0.613\end{array}$ & $\begin{array}{r}0.42(0.10- \\
1.82) \\
p=0.245\end{array}$ \\
\hline
\end{tabular}

* Denominators vary due to missing values.

† Univariate regression analyses with $95 \% \mathrm{Cl}$ for the associations between the analyzed groups.

${ }^{\S}$ Multivariate logistic regression analyses with $95 \% \mathrm{Cl}$, adjusting for the covariates (parity, maternal age, BMl, smoking habits, maternal origin). 
Table 5 Cross-sectional population post-intervention: Experiences with use of a kick chart $(\mathbf{N}=235)^{*}$

\begin{tabular}{|c|c|c|c|c|c|}
\hline $\begin{array}{l}\text { Maternal experiences with use } \\
\text { of a kick cart }\end{array}$ & n (\%) & $\begin{array}{r}\text { Recalled receipt of information about } \\
\text { HOW to use the kick chart } \\
n=119(63.3 \%) \\
\text { OR }(95 \% \mathrm{Cl}) \dagger\end{array}$ & $\begin{array}{r}P \\
\text { value }\end{array}$ & $\begin{array}{r}\text { Recalled receipt of information about } \\
\text { WHY use the kick chart } \\
n=121(66.5 \%) \\
\text { OR }(95 \% \mathrm{Cl}) \dagger\end{array}$ & $\begin{array}{r}P \\
\text { value }\end{array}$ \\
\hline $\begin{array}{l}\text { Kick counting was time- } \\
\text { consuming }\end{array}$ & $\begin{array}{r}97 \\
(41.3)\end{array}$ & $0.3(0.1-0.7)$ & 0.003 & $0.4(0.2-0.9)$ & 0.039 \\
\hline $\begin{array}{l}\text { Kick counting stimulated to "get } \\
\text { to know" the baby }\end{array}$ & $\begin{array}{r}71 \\
(30.2)\end{array}$ & $2.2(0.9-5.4)$ & 0.099 & $1.7(0.7-4.3)$ & 0.251 \\
\hline $\begin{array}{l}\text { Appreciated the visual } \\
\text { presentation of the fetal activity }\end{array}$ & $\begin{array}{r}74 \\
(31.5)\end{array}$ & $3.3(1.3-8.4)$ & 0.011 & $1.9(0.7-4.9)$ & 0.189 \\
\hline $\begin{array}{l}\text { Kick counting induced too much } \\
\text { focus on fetal activity }\end{array}$ & $\begin{array}{r}47 \\
(20.0)\end{array}$ & $1.2(0.5-3.0)$ & 0.568 & $0.8(0.4-1.9)$ & 0.727 \\
\hline
\end{tabular}

* Denominators vary due to missing values.

† Univariate regression analyses with $95 \% \mathrm{Cl}$ for the associations between the analyzed groups. Reference groups: women who did not recall receipt of information about how to and why use of a kick chart respectively.

decrease in or absence of fetal movement. This may have contributed to the decreased stillbirth rate among primiparous women. The importance of recognizing DFM for pregnancy outcomes is indisputable $[2,4,22]$, and identification of risk is one of the main goals for antenatal care [47]. Women were advised to contact their health care provider for concerns about DFM regardless of reaching in any specific fetal movement rate threshold. The advice on focused counting and the suggested "alarm limits" $[7,11]$ when women were in doubt about the presence of DFM in addition to the advice about their perceptions may have contributed to a reduction in excessive delay in reporting DFM.

A similar proportion (75\%) of women recalled having received information in the baseline and the intervention. Thus, this provides support to the effectiveness of the information to improve maternal self-screening of DFM which was more explicit than the previous information [18] and emphasized maternal assessment of fetal activity according to the activity pattern for her own child [9]. This was reflected in the mothers' reasoning for concern in the post-intervention. Women in the post-implementation period reported concerns related to the activity level earlier in the pregnancy more often and were more confident that their perception of DFM was the true reason for being concerned.

\section{Overweight mothers - higher awareness, more concerns, but not improved pregnancy outcomes}

Being overweight increases the risk of not perceiving DFM (Tveit et al, submitted 2009). However, it is unknown whether reduced perception of fetal movements among overweight women is due to higher risk of a true decrease in fetal movement or to a lower ability to perceive fetal activity [27]. In the post-intervention period, overweight women described higher awareness of fetal activity, they more frequently reported concerns of DFM and presented at the hospital during the night more frequently. However, no difference was shown in the excessive delay in reporting of decreased or absent fetal movement or in the stillbirth rate among these mothers.

\section{Mothers of non-Western origin - less access to information}

In the non-Western population the intervention was not associated with changes in maternal behavior or the stillbirth rates. Non-Western women had three times increased risk of low awareness of fetal activity when compared to the Western mothers, and were shown to have the lowest rates of receiving the information about expected fetal activity, in spite of available information brochures in the most common foreign languages in the area. This may be due to the presentation of the information not adequately meeting their needs or to cultural differences in risk orientation [48]. Communication problems between non-Western women and health care providers have been identified as a risk factor for adverse pregnancy outcomes $[29,48]$. This confirms the need for a greater focus on providing culturally appropriate information which is written at an appropriate level to ensure comprehensive uptake particularly for those women at increased risk [49].

The population non-Western women in our study were mainly from low-income countries and a wide variation in cultures was represented. Minority or marginalized women in a high-income country do not appear to display a "healthism" approach to their lives [50]; normative assumptions in antenatal guidelines do not apply. This may be in part due to a lack of trust in caregivers among minority women in Western countries [50,51], the authoritative source often are their husband [51] or their mother [52], instead of the health care services. While printed educational materials are widely used to improve knowledge, awareness and attitudes, especially in developed countries, other methods for information 
and education may be needed for cultural minority groups. The impact of life style choices and compliance to recommendations from health providers may be higher if role models and authoritative sources, such as the husband and/or mother, are involved in the antenatal care. Further research is needed on appropriate methods to change health seeking behavior in pregnancy, including DFM, for non-Western women in our setting.

Fetal movement counting associated with well-being and safety

While the majority of women chose not to use a kick chart, its use was associated with less maternal concerns, as well as a reduced risk of being examined in hospital because of DFM. Satisfaction with the information about the rationale for fetal monitoring and the technique of recording were associated with more frequent use of a kick chart and increased the mothers' assessment that a kick chart was important and useful. Effective communication specific for each woman's need and encouragement by a consistent healthcare professional have been identified as the key factor for high compliance for use of a kick chart $[6,31,53]$.

Many health professionals do not to recommend a FMC in their low-risk patients because they fear it will cause increased maternal concern and anxiety [54], as well as increased unnecessary consultations and/or interventions $[7,55]$. The current study was not a study to evaluate the use of kick chart per se. Nevertheless, it is important to notice that use of a kick chart was not associated with increased concerns or more frequent consultations in the hospital. It seemed to be "safe" with regard to maternal well-being and use of health resources. We have no evidence that FMC with specific alarm limits are preferable or superior to subjective maternal opinion. However, previous reports also indicate that the use of a kick chart does not cause anxiety or other adverse psychological effects $[54,56,57]$. Further research is needed, both in low- and high-risk populations $[4,12]$.

\section{Methodological considerations}

The true effect of such an intervention may be better estimated using a randomized trial methodology. However, in a quality improvement setting like ours, a before-and-after study design was chosen. There are potential problems in using RCT to test the effect of information within the same population due to the likelihood of contamination. While the before-andafter study design may overestimate the true effect, the prospective nature of this study may limit this effect. Additional methodological considerations are presented in the article from the other part of the quality improvement, the clinical management of DFM pregnancies [30]. Potential recall bias and the validity of the cross-sectional questionnaire have been discussed elsewhere [10].

While these findings are encouraging, caution in its interpretation is warranted due to limitations of the design employed in this quality improvement project; the implemented solutions were based on the local existing imperfections found by prior data collections of quality indicators. The results may thus not be directly transferable to other populations. Yet, reports from a variety of locations suggest that significant variability in the information given to expecting women is a wide-spread quality issue in obstetric care $[15,22,23,58]$.

\section{Conclusions}

Uniform information about fetal activity provided to pregnant women was associated with a reduction in the number of primiparous women who delayed reporting of DFM and reduced stillbirth rates for primiparous women reporting DFM. The information did not appear to increase maternal concerns or frequency of consultations. While these findings are encouraging, caution in its interpretation is warranted due to limitations of the design employed in this quality improvement project; the implemented solutions were based on the local issues identified by prior quality assurance studies. Further studies replicating these findings are required. A clearer definition of DFM is needed.

Additional file 1: Kick Count. Kicks Count brochure, Norwegian version.
A brochure of information aiming to increase maternal awareness and
vigilance to significant decreases in fetal activity, and to aid health
promoting behavior. The brochure was provided as a part of the routine
information given to women at the standard ultrasound assessment at
17-19 weeks in Norway as a part of the quality improvement
intervention.
Click here for file
[http://www.biomedcentral.com/content/supplementary/1756-0500-3-2-
S1.PDF ]
Additional file 2: Kick Count. Kicks Count brochure, English version. A
brochure of information aiming to increase maternal awareness and
vigilance to significant decreases in fetal activity, and to aid health
promoting behavior. The brochure was provided as a part of the routine
information given to women at the standard ultrasound assessment at
17-19 weeks in Norway as a part of the quality improvement
intervention.
Click here for file
[http://www.biomedcentral.com/content/supplementary/1756-0500-3-2-
S2.PDF]
Additional file 3: Kick Count. Kicks Count brochure, Urdu version. A
brochure of information aiming to increase maternal awareness and
vigilance to significant decreases in fetal activity, and to aid health
promoting behavior. The brochure was provided as a part of the routine
information given to women at the standard ultrasound assessment at
17-19 weeks in Norway as a part of the quality improvement
intervention.
Click here for file
[http://www.biomedcentral.com/content/supplementary/1756-0500-3-2-
S3.PDF]

Additional file 1: Kick Count. Kicks Count brochure, Norwegian version. A brochure of information aiming to increase maternal awareness and promoting behavior. The brochure was provided as a part of the routine information given to women at the standard ultrasound assessment at S1.PDF ]

brochure of information aiming to increase maternal an vigilance to significant decreases in fetal activity, and to aid health promoting behavior. The brochure was provided as a part of the routine 17-19 weeks in Norway as a part of the quality improvement intervention

Click here for file

[http://www.biomedcentral.com/content/supplementary/1756-0500-3-2

brochure of information aiming to increase maternal awareness and vigilance to significant decreases in fetal activity, and to aid health promoting behavior. The brochure was provided as a part of the routine 17-19 weeks in Norway as a part of the quality improvement intervention.

[http://www.biomedcentral.com/content/supplementary/1756-0500-3-2 
Additional file 4: Kick Count. Kicks Count brochure, Somali version. A brochure of information aiming to increase maternal awareness and vigilance to significant decreases in fetal activity, and to aid health promoting behavior. The brochure was provided as a part of the routine information given to women at the standard ultrasound assessment at 17-19 weeks in Norway as a part of the quality improvement intervention.

Click here for file

[http://www.biomedcentral.com/content/supplementary/1756-0500-3-2S4.PDF ]

Additional file 5: Kick Count. Kicks Count brochure, Turkish version. A brochure of information aiming to increase maternal awareness and vigilance to significant decreases in fetal activity, and to aid health promoting behavior. The brochure was provided as a part of the routine information given to women at the standard ultrasound assessment at 17-19 weeks in Norway as a part of the quality improvement intervention.

Click here for file

[http://www.biomedcentral.com/content/supplementary/1756-0500-3-2S5.PDF ]

Additional file 6: Kick Count. Kicks Count brochure, Arabic version. A brochure of information aiming to increase maternal awareness and vigilance to significant decreases in fetal activity, and to aid health promoting behavior. The brochure was provided as a part of the routine information given to women at the standard ultrasound assessment at 17-19 weeks in Norway as a part of the quality improvement intervention.

Click here for file

[http://www.biomedcentral.com/content/supplementary/1756-0500-3-2S6.PDF ]

Additional file 7: Example of use of a kick chart. An example of a kick chart used by a women participating in the study

Click here for file

[http://www.biomedcentral.com/content/supplementary/1756-0500-3-2S7.PDF ]

\section{Acknowledgements}

We want to express a special gratitude to all the participating hospitals and their coordinators for collecting the presented material: Live Grindaker Ask, Ingrid Borthen, Cecilie Bryn, Elisabeth Hals, Nora Pran Halvorsen, Kari Haugødegård, Elisabeth Heiberg, Lina Herstad, Ann Holstvoll, Berit Jacobsen, Åse Kari Kringlåk, Kristine Lem, Hege Lundring, Hårek Lysberg, Zelalem Mengistu, Mette Nordbø, Cecilie Nordklev, Lill Nyfløt, Richard Olsson, IngerLise Paulsen, Torill O'Reilly, Kjersti Rimstad, Åse Karin Rondestveit, Siri Skrøppa, Ruth Svarstad, Asbjørn Myren Svendsen, Torgrim Sørnes, Ursula Teufel, Ameli Trope, Ellen-Sofie Trovik, Christin Wendt, Stian Westad, and Line Aaby,

This work has been supported by grants from The Norwegian Research Council, The Norwegian SIDS and Stillbirth Society, The Norwegian Women's Public Health Association, The Norwegian Medical Association, Unger Vetlesen Medical Foundation, Blix Foundation, Odd Fellow Foundation, Fulbright Foundation, American Women's Club of Oslo and Medinnova, The Norwegian Foundation for Health and Rehabilitation and National Resource Centre for Women's Health, Rikshospitalet University Hospital. The funding sources had no involvement in the authors' work.

\section{Author details}

${ }^{1}$ Norwegian Institute of Public Health, Division of Epidemiology, Oslo, Norway. ${ }^{2}$ Akershus University College, Lillestrøm, Norway. ${ }^{3}$ Department of Obstetrics and Gynecology, Centre for Perinatal Research, Rikshospitalet University Hospital, Oslo, Norway. ${ }^{4}$ University of Oslo, Medical faculty, Norway. ${ }^{5}$ Department of Obstetrics and Gynecology, University of Queensland, Mater Mothers' Hospital, South Brisbane, Australia. ${ }^{6}$ Brigham and Women's Hospital, Division of Maternal-Fetal Medicine, Harvard Medical School, Boston, MA and Harvard Medical Associates, Wellesley, MA, USA. ${ }^{7}$ Institutes for Clinical Medicine, Section for Gynecology and Obstetrics, and University of Bergen, Norway.

\section{Authors' contributions}

ES: Design of the study, data collection, analysis, interpretation of data, writing and finalizing the manuscript. JVHT: Design of the study, data collection, interpretation of data and revising the manuscript. VF: Design of the study, interpretation of data, writing and revising the manuscript. BSP: Design of the study, interpretation of data and revising the manuscript. PEB: Design of the study. RF: Design of the study, interpretation of data and revising the manuscript. JFF: Design of the study, analysis, interpretation of data, writing and revising the manuscript.

All authors have approved the final version of the manuscript.

\section{Competing interests}

The authors declare that they have no competing interests.

Received: 3 September 2009

Accepted: 4 January 2010 Published: 4 January 2010

\section{References}

1. Sinha $D$, et al: Obstetric outcome in women complaining of reduced fetal movements. J Obstet Gynaecol 2007, 27:41-43.

2. Olesen AG, Svare JA: Decreased fetal movements: background, assessment, and clinical management. Acta Obstet Gynecol Scand 2004 83:818-26.

3. Heazell $A E$, Sumathi $G M$, Bhatti NR: What investigation is appropriate following maternal perception of reduced fetal movements?. J Obstet Gynaecol 2005, 25:648-650.

4. Mangesi L, Hofmeyr GJ: Fetal movement counting for assessment of fetal wellbeing (Review). John Wiley \& Sons, Ltd, 12007.

5. Frøen JF, others: Risk factors for sudden intrauterine unexplained death: Epidemiologic characteristics of singleton cases in Oslo, Norway, 19861995. Am J Obstet Gynecol 2001, 184:694-702.

6. Grant A, others: Routine formal fetal movement counting and risk of antepartum late death in normally formed singletons. Lancet 1989, 2:345349

7. Heazell AEP, Frøen JF: Methods of fetal movement counting and the detection of fetal compromise. J Obstet Gynaecol 2008, 28:147-154.

8. Frøen JF, et al: Fetal movement assessment. Semin Perinatol 2008, 32:243246.

9. Groome LD, Swiber MJ, Holland SB, Bentz LS, Atterbury JL, Trimm RF 3rd: Spontaneous motor activity in the perinatal infant before and after birth, stability in individual differences. Dev Psychobiol. 1999, 35(1):25-34

10. Saastad E, Ahlborg T, Frøen JF: Low maternal awareness of fetal movement is associated with small for gestational age infants. $J$ Midwifery Womens Health 2008, 53:345-352.

11. Moore TR, Piacquadio K: A Prospective Evaluation of Fetal Movement Screening to Reduce the Incidence of Antepartum Fetal Death. Am J Obstet Gynecol 1989, 160:1075-1080.

12. Haws RA, et al: Reducing stillbirths: screening and monitoring during pregnancy and labour. BMC Pregnancy Childbirth. 2009, 9(Suppl 1):S5.

13. Neldam S: Fetal movements as an indicator of fetal well-being. Dan Med Bull 1983, 30:274-8.

14. Berbey R, Manduley P, De-Vigil G: Counting fetal movements as a universal test for fetal wellbeing. International Journal of Gynaecology \& Obstetrics 2001, 74:293-295

15. Heazell $A E$, et al: Midwives' and obstetricians' knowledge and management of women presenting with decreased fetal movements. Acta Obstet Gynecol Scand. 2008, 87(3):331-339.

16. Frøen JF: A kick from within-fetal movement counting and the cancelled progress in antenatal care. J Perinat Med 2004, 32:13-24

17. Guidelines for perinatal care Elk Grove Village, IL \& Washington DC: American Academy of Pediatrics, The American College of Obstetricians and Gynecologists, 52002.

18. Sosial- og helsedirektoratet: Retningslinjer for svangerskapsomsorgen [Guidelines for antenatal care]. [Norwegian] Oslo: Sosial- og Helsedirektoratet 2005.

19. RCOG: Antenatal care - routine care for the healthy pregnant woman London: RCOG Press 2003.

20. Kohner N, others: The Pregnancy Book London: The Department of Health UK, 72007.

21. Sergent $F$, et al: [Decreased fetal movements in the third trimester: what to do?]. Gynecol Obstet Fertil 2005, 33:861-869. 
22. Frøen JF: [Clinical practice variation in reduced fetal movements]. Tidsskr Nor Laegeforen 2005, 125:2631-2634.

23. Flenady V, MacPhail J, Gardener G, Chadha Y, Mahomed K, Egan S, Heazell AE, Fretts RC, Frøen JF: Management of decreased fetal movements in Australia and New Zealand: A survey of practice. Perinatal Society of Australia and New Zealand 9th Annual Congress 2006.

24. Fretts R, Frøen JF, Cavanagh E, Reynolds D: A prospective study of pregnancies with Decreased Fetal Movement. 3rd Annual Conference of the International Stillbirth Alliance 2007.

25. Safe Practices for Better Healthcare: A Consensus Report. 2003 Washington, DC: The National Quality Forum 2009.

26. Fretts RC: Etiology and prevention of stillbirth. Am J Obstet Gynecol 2005, 193:1923-1935.

27. Sebire NJ, et al: Maternal obesity and pregnancy outcome: a study of 287213 pregnancies in London. International Journal of Obesity \& Related Metabolic Disorders 2001, 25:1175-82.

28. Coppens M: Computerized analysis of acute and chronic changes in fetal heart rate variation and fetal activity in association with maternal smoking. Am J Obstet Gynecol 2001, 185:421-426.

29. Saastad E, Vangen S, Frøen JF: Suboptimal care in stillbirths - a retrospective audit study. Acta Obstet Gynecol Scand 2007, 86:444-450.

30. Tveit JV, et al: Reduction of late stillbirth with the introduction of fetal movement information and guidelines - a clinical quality improvement. BMC Pregnancy and Childbirth 2009, 9:32.

31. Velazquez MD, Rayburn WF: Antenatal Evaluation of the Fetus Using Fetal Movement Monitoring. Clin Obstet Gynecol 2002, 45:993-1004.

32. Cito $G$, et al: Maternal position during non-stress test and fetal heart rate patterns. Acta Obstet Gynecol Scand 2005, 84:335-338.

33. Graca $L M$, et al: Acute effects of maternal cigarette smoking on fetal heart rate and fetal body movements felt by the mother. J Perinat Med 1991, 19:385-390.

34. American Academy of Pediatrics, The American College of Obstetricians and Gynecologists: Guidelines for perinatal care Washington, DC: AAP and ACOG, 52002

35. Frøen JF, et al: Management of Decreased Fetal Movements. Semin Perinatol 2008, 32:307-311.

36. Pearson JF: Fetal movement recording: a guide to fetal well-being. Nurs Times 1979, 75:1639-1641.

37. Christensen FC, Olson K, Rayburn WF: Cross-over trial comparing maternal acceptance of two fetal movement charts. Journal of Maternal-Fetal \& Neonatal Medicine 2003, 14:118-122.

38. Gomez $L M$, et al: Compliance with a fetal movement chart by high-risk obstetric patients in a Peruvian hospital. Am J Perinatol 2007, 24:89-93.

39. The Medical Birth Registry of Norway 2007http://www.fhi.no/eway/default. aspx?pid=233\&trg=MainArea 5661\&MainArea_5661=5665:0:15.3278:1:0:0: 0

40. Sadovsky $E$, et al: The definition and the significance of decreased fetal movements. Acta Obstet Gynecol Scand 1983, 62:409-413.

41. Harrington $\mathrm{K}$, et al: Obstetric outcome in women who present with a reduction in fetal movements in the third trimester of pregnancy. $J$ Perinat Med 1998, 26:77-82

42. Gardosi J: Customized fetal growth standards: rationale and clinical application. Seminars in Perinatology 2004, 28:33-40.

43. Hosmer D, Lemeshow S: Applied Logistic Regression John Wiley \& Sons Inc, 2 2000.

44. Deave T, Johnson $D$, Ingram J: Transition to parenthood: the needs of parents in pregnancy and early parenthood. BMC Pregnancy and Childbirth 2008, 8:30

45. Farmer AP, et al: Printed educational materials: effects on professional practice and health care outcomes. Cochrane Database of Systematic Reviews 2008, CD004398.

46. Grimshaw JM, et al: Effectiveness and efficiency of guideline dissemination and implementation strategies. Health Technology Assessment (Winchester, England) 2001, 8:iii-iiv.

47. Chalmers B, Mangiaterra V, Porter R: WHO principles of perinatal care: the essential antenatal, perinatal, and postpartum care course. Birth 2001 28:202-207.

48. Essen B, others: Are some perinatal deaths in immigrant groups linked to suboptimal perinatal care services?. BJOG: an International Journal of Obstetrics \& Gynaecology 2002, 109:677-682.

49. Freda MC: Issues in patient education. Journal of Midwifery \& Women's Health 2004, 49:203-209.
50. Downe $S$, et al: 'Weighing up and balancing out': a meta-synthesis of barriers to antenatal care for marginalised women in high-income countries. BJOG: An International Journal of Obstetrics and Gynaecology 2009, 116:518-529.

51. Ny P, et al: Middle Eastern mothers in Sweden, their experiences of the maternal health service and their partner's involvement. Reproductive Health 2007, 4:9.

52. Burton LM: Age norms, the timing of family role transitions, and intergenerational caregiving among aging African American women. The Gerontologist 1996, 36:199.

53. Kuwata T, et al: Establishing a reference value for the frequency of fetal movements using modified "count to 10" method. J Obstet Gynaecol Res 2008, 34:318-323.

54. Hill-Smith I: Professional and patient perspectives of NICE guidelines to abandon maternal monitoring of fetal movements. Br J Gen Pract 2004, 54:858-861.

55. Flenady V, Gardener G, MacPhail J, Chadha Y, King J, Cole S, McCowan LF, Frøen JF: Fetal Movement Monitoring: Practice in Australia and New Zealand. The Perinatal Society of Australia and New Zealand, 9th Annual Congress, Perth 2006.

56. Liston RM, Bloom K, Zimmer P: The psychological effects of counting fetal movements. Birth 1994, 21:135-140.

57. Mikhail MS, et al: The effect of fetal movement counting on maternal attachment to fetus. American Journal of Obstetrics \& Gynecology 1991, 165:988-991.

58. Saastad E, Frøen JF: [Reduced fetal movements-clinical management, recommendations and information]. Tidsskr Nor Laegeforen 2005, 125:2627-2630

doi:10.1186/1756-0500-3-2

Cite this article as: Saastad et al: Implementation of uniform information on fetal movement in a Norwegian population reduced delayed reporting of decreased fetal movement and stillbirths in primiparous women - a clinical quality improvement. BMC Research Notes 2010 3:2.

\section{Submit your next manuscript to BioMed Central and take full advantage of:}

- Convenient online submission

- Thorough peer review

- No space constraints or color figure charges

- Immediate publication on acceptance

- Inclusion in PubMed, CAS, Scopus and Google Scholar

- Research which is freely available for redistribution 